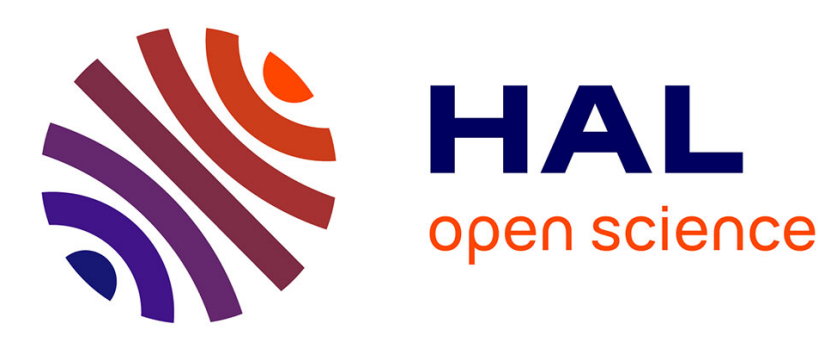

\title{
Introduction to Kernel Methods: Classification of Multivariate Data
}

\author{
Mathieu Fauvel
}

\section{To cite this version:}

Mathieu Fauvel. Introduction to Kernel Methods: Classification of Multivariate Data. EAS Publications Series, 2016, 77, pp.171-193. 10.1051/eas/1677008 . hal-02630105

\section{HAL Id: hal-02630105 \\ https://hal.inrae.fr/hal-02630105}

Submitted on 27 May 2020

HAL is a multi-disciplinary open access archive for the deposit and dissemination of scientific research documents, whether they are published or not. The documents may come from teaching and research institutions in France or abroad, or from public or private research centers.
L'archive ouverte pluridisciplinaire HAL, est destinée au dépôt et à la diffusion de documents scientifiques de niveau recherche, publiés ou non, émanant des établissements d'enseignement et de recherche français ou étrangers, des laboratoires publics ou privés. 


\title{
Introduction to Kernel Methods \\ Classification of multivariate data
}

\author{
Mathieu Fauvel
}

$<2015-10-13$ Tue $>$ 


\section{Outline}

Introductory example

Linear case

Non linear case

Concluding remarks

Kernel function

Positive semi-definite kernels

Some kernels

Concluding remarks

Kernel K-NN

Support Vectors Machines

Learn from data

Linear SVM

Non linear SVM

Fitting the hyperparameters

Multiclass SVM

Classification of hyperspectral data 
Inner product

An inner product is a map $\langle., .\rangle_{\mathcal{X}}: \mathcal{X} \times \mathcal{X} \rightarrow \mathcal{K}$ satisfying the following axioms:

- Symmetry: $\langle\mathbf{x}, \mathbf{y}\rangle_{\mathcal{X}}=\langle\mathbf{y}, \mathbf{x}\rangle_{\mathcal{X}}$

- Bilinearity:

$\langle a \mathbf{x}+b \mathbf{y}, c \mathbf{z}+d \mathbf{w}\rangle_{\mathcal{X}}=a c\langle\mathbf{x}, \mathbf{z}\rangle_{\mathcal{X}}+a d\langle\mathbf{x}, \mathbf{w}\rangle_{\mathcal{X}}+b c\langle\mathbf{y}, \mathbf{z}\rangle_{\mathcal{X}}+b d\langle\mathbf{y}, \mathbf{w}\rangle_{\mathcal{X}}$

- Non-negativity: $\langle\mathbf{x}, \mathbf{x}\rangle_{\mathcal{X}} \geq 0$

- Positive definiteness: $\langle\mathbf{x}, \mathbf{x}\rangle_{\mathcal{X}}=0 \Leftrightarrow \mathbf{x}=\mathbf{0}$

The standard inner product in the Euclidean space, $\mathrm{x} \in \mathbb{R}^{d}$ and $d \in \mathbb{N}$, is called the dot product: $\langle\mathbf{x}, \mathbf{y}\rangle_{\mathbb{R}^{n}}=\sum_{i=1}^{n} x_{i} y_{i}$. 
Introductory example

Linear case

Non linear case

Concluding remarks

Kernel function

Positive semi-definite kernels

Some kernels

Concluding remarks

Kernel K-NN

Support Vectors Machines

Learn from data

Linear SVM

Non linear SVM

Fitting the hyperparameters

Multiclass SVM 
Introductory example

Linear case

Non linear case

Concluding remarks

Kernel function

Positive semi-definite kernels

Some kernels

Concluding remarks

Kernel K-NN

Support Vectors Machines

Learn from data

Linear SVM

Non linear SVM

Fitting the hyperparameters

Multiclass SVM

Classification of hyperspectral data 


\section{Toy data set}

Suppose we want to classify the following data

$$
\mathcal{S}=\left\{\left(\mathbf{x}_{1}, y_{1}\right), \ldots,\left(\mathbf{x}_{n}, y_{n}\right)\right\},\left(\mathbf{x}_{i}, y_{i}\right) \in \mathbb{R}^{2} \times\{ \pm 1\}
$$

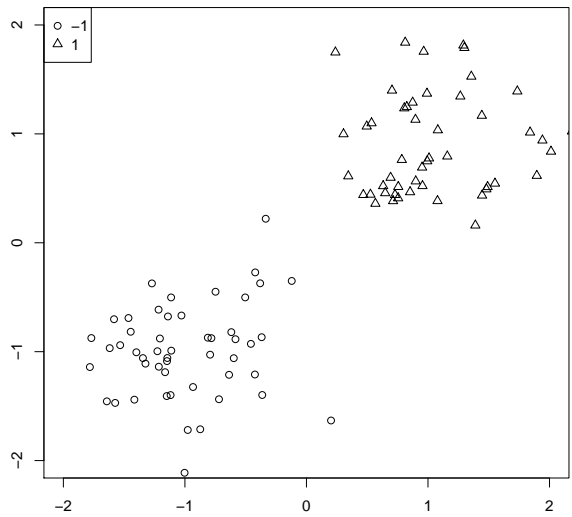




\section{Simple classifier:}

- Decision rule: assigns a new sample $\mathbf{x}$ to the class whose mean is closer to $\mathrm{x}$.

$$
f(\mathbf{x})=\operatorname{sgn}\left(\left\|\mu_{-1}-\mathbf{x}\right\|^{2}-\left\|\mu_{1}-\mathbf{x}\right\|^{2}\right) .
$$

- Equation (1) can be written in the following way

$$
f(\mathbf{x})=\operatorname{sgn}(\langle\mathbf{w}, \mathbf{x}\rangle+b) .
$$

- Identify $\mathbf{w}$ and $b$. Tips: $\left\|\mu_{-1}-\mathbf{x}\right\|^{2}=\left\langle\mu_{-1}-\mathbf{x}, \mu_{-1}-\mathbf{x}\right\rangle$ and

$$
\mu_{-1}=\frac{1}{m_{-1}} \sum_{i=1}^{m_{-1}} \mathbf{x}_{i}
$$




\section{Solution $1 / 3$}

$$
\begin{aligned}
\left\|\mu_{1}-\mathbf{x}\right\|^{2} & =\left\langle\frac{1}{m_{1}} \sum_{i=\mathbf{1}}^{m_{1}} \mathbf{x}_{i}-\mathbf{x}, \frac{1}{m_{1}} \sum_{i=\mathbf{1}}^{m_{\mathbf{1}}} \mathbf{x}_{i}-\mathbf{x}\right\rangle \\
& =\left\langle\frac{1}{m_{1}} \sum_{i=\mathbf{1}}^{m_{\mathbf{1}}} \mathbf{x}_{i}, \frac{1}{m_{1}} \sum_{i=\mathbf{1}}^{m_{\mathbf{1}}} \mathbf{x}_{i}\right\rangle+\langle\mathbf{x}, \mathbf{x}\rangle-2\left\langle\frac{1}{m_{1}} \sum_{i=\mathbf{1}}^{m_{\mathbf{1}}} \mathbf{x}_{i}, \mathbf{x}\right\rangle \\
& =\frac{1}{m_{1}^{2}} \sum_{\substack{i=\mathbf{1} \\
k=1}}^{m_{\mathbf{1}}}\left\langle\mathbf{x}_{i}, \mathbf{x}_{k}\right\rangle+\langle\mathbf{x}, \mathbf{x}\rangle-2\left\langle\frac{1}{m_{1}} \sum_{i=1}^{m_{1}} \mathbf{x}_{i}, \mathbf{x}\right\rangle \\
\left\|\mu_{-1}-\mathbf{x}\right\|^{2} & =\frac{1}{m_{-1}^{2}} \sum_{\substack{j=\mathbf{1} \\
l=\mathbf{1}}}^{m_{-1}}\left\langle\mathbf{x}_{j}, \mathbf{x}_{l}\right\rangle+\langle\mathbf{x}, \mathbf{x}\rangle-2\left\langle\frac{1}{m_{-1}} \sum_{j=\mathbf{1}}^{m_{-1}} \mathbf{x}_{j}, \mathbf{x}\right\rangle
\end{aligned}
$$

Plugging (2) and (3) into (1) leads to

$$
\langle\mathbf{w}, \mathbf{x}\rangle+b=\left\langle 2\left(\frac{1}{m_{1}} \sum_{i=1}^{m_{1}} \mathbf{x}_{i}-\frac{1}{m_{-1}} \sum_{j=1}^{m_{-1}} \mathbf{x}_{j}\right), \mathbf{x}\right\rangle-\frac{1}{m_{1}^{2}} \sum_{\substack{i=1 \\ k=1}}^{m_{1}}\left\langle\mathbf{x}_{i}, \mathbf{x}_{k}\right\rangle+\frac{1}{m_{-1}^{2}} \sum_{\substack{j=1 \\ l=1}}^{m_{-1}}\left\langle\mathbf{x}_{j}, \mathbf{x}_{l}\right\rangle
$$


Solution $2 / 3$

$$
\begin{aligned}
\mathbf{w} & =2 \sum_{i=\mathbf{1}}^{m_{\mathbf{1}}+m_{-1}} \alpha_{i} y_{i} \mathbf{x}_{i} \\
y_{i} & =1 \text { or }-1 \\
\alpha_{i} & =\frac{1}{m_{1}} \text { or } \frac{1}{m_{-1}} \\
b & =-\frac{1}{m_{1}^{2}} \sum_{\substack{i=1 \\
k=1}}^{m_{1}}\left\langle\mathbf{x}_{i}, \mathbf{x}_{k}\right\rangle+\frac{1}{m_{-1}^{2}} \sum_{\substack{j=1 \\
l=1}}^{m_{-1}}\left\langle\mathbf{x}_{j}, \mathbf{x}_{l}\right\rangle
\end{aligned}
$$




\section{Solution $3 / 3$}

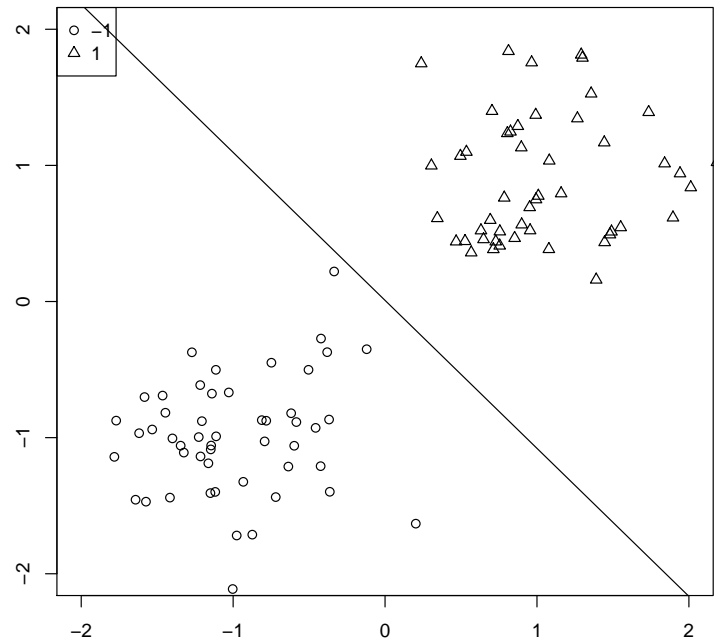


Introductory example

Linear case

\section{Non linear case}

Concluding remarks

Kernel function

\section{Positive semi-definite kernels \\ Some kernels \\ Concluding remarks}

Kernel K-NN

Support Vectors Machines

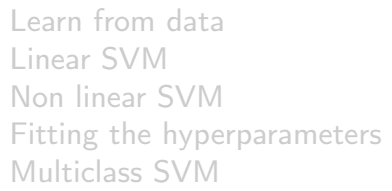

Classification of hyperspectral data 


\section{Another toy data set}

- Now the data are distributed a bit differently:

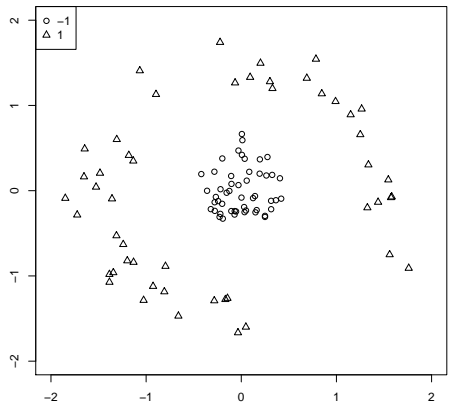

- However, if we can find a feature space where the data are linearly separable, it can still be applied.

- Questions:

1. Find a feature space where the data are linearly separable.

2. Try to write the dot product in the feature space in terms of input space variables. 


\section{Feature space}

Two simple feature spaces are possible:

1. Projection in the polar domain

$$
\begin{aligned}
& \rho=\mathbf{x}_{1}^{2}+\mathbf{x}_{2}^{2} \\
& \theta=\arctan \left(\frac{\mathbf{x}_{2}}{\mathbf{x}_{1}}\right)
\end{aligned}
$$

2. Projection in the space of monomials of order 2 .

$$
\begin{aligned}
\phi: \mathbb{R}^{2} & \rightarrow \mathbb{R}^{3} \\
\mathbf{x} & \mapsto \phi(\mathbf{x}) \\
\left(\mathbf{x}_{1}, \mathbf{x}_{2}\right) & \mapsto\left(\mathbf{x}_{1}^{2}, \mathbf{x}_{2}^{2}, \sqrt{2} \mathbf{x}_{1} \mathbf{x}_{2}\right)
\end{aligned}
$$


Feature space associated to monomials of order 2

- In $\mathbb{R}^{3}$, the inner product can be expressed as

$$
\begin{aligned}
\left\langle\phi(\mathbf{x}), \phi\left(\mathbf{x}^{\prime}\right)\right\rangle_{\mathbb{R}^{3}} & =\sum_{i=\mathbf{1}}^{3} \phi(\mathbf{x})_{i} \phi\left(\mathbf{x}^{\prime}\right)_{i} \\
& =\phi(\mathbf{x})_{1} \phi\left(\mathbf{x}^{\prime}\right)_{1}+\phi(\mathbf{x})_{2} \phi\left(\mathbf{x}^{\prime}\right)_{2}+\phi(\mathbf{x})_{3} \phi\left(\mathbf{x}^{\prime}\right)_{3} \\
& =\mathbf{x}_{1}^{2} \mathbf{x}_{1}^{\prime}{ }_{1}+\mathbf{x}_{2}^{2} \mathbf{x}^{\prime 2}{ }_{2}+2 \mathbf{x}_{1} \mathbf{x}_{2} \mathbf{x}^{\prime}{ }_{1} \mathbf{x}^{\prime}{ }_{2} \\
& =\left(\mathbf{x}_{1} \mathbf{x}_{1}^{\prime}+\mathbf{x}_{2} \mathbf{x}^{\prime}{ }_{2}\right)^{2} \\
& =\left\langle\mathbf{x}, \mathbf{x}^{\prime}\right\rangle_{\mathbb{R}^{2}}^{2} \\
& =k\left(\mathbf{x}, \mathbf{x}^{\prime}\right) .
\end{aligned}
$$

- The decision rule can be written in the input space thanks to the function $k$.

$$
f(\mathbf{x})=2 \sum_{i=1}^{m_{1}+m_{-1}} \alpha_{i} y_{i} k\left(\mathbf{x}_{i}, \mathbf{x}\right)-\frac{1}{m_{1}^{2}} \sum_{\substack{i=1 \\ k=1}}^{m_{1}} k\left(\mathbf{x}_{i}, \mathbf{x}_{k}\right)+\frac{1}{m_{-1}^{2}} \sum_{\substack{j=1 \\ l=1}}^{m_{-1}} k\left(\mathbf{x}_{j}, \mathbf{x}_{l}\right)
$$




\section{Non linear decision function}

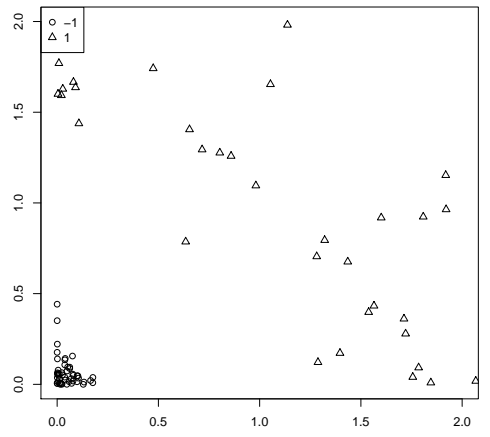

Feature space $\left(x_{1}^{2}, x_{2}^{2}\right)$

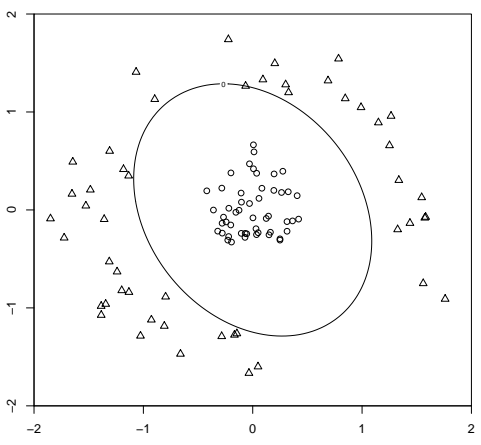

Separating function 
Introductory example

Linear case

Non linear case

Concluding remarks

Kernel function

Positive semi-definite kernels

Some kernels

Concluding remarks

Kernel K-NN

Support Vectors Machines

Learn from data

Linear SVM

Non linear SVM

Fitting the hyperparameters

Multiclass SVM

Classification of hyperspectral data 


\section{Conclusion}

- A linear algorithm can be turned to a non linear one, simply by exchanging the dot product by an appropriate function.

- This function has to be equivalent to a dot product in a feature space.

- It is called a kernel function or just kernel.

- What are the properties of kernel? 


\section{Linear case \\ Non linear case \\ Concluding remarks}

Kernel function

Positive semi-definite kernels

Some kernels

Concluding remarks

Kernel K-NN

Support Vectors Machines

Learn from data

Linear SVM

Non linear SVM

Fitting the hyperparameters

Multiclass SVM 
Introductory example

Linear case

Non linear case

Concluding remarks

Kernel function

Positive semi-definite kernels

Some kernels

Concluding remarks

Kernel K-NN

Support Vectors Machines

Learn from data

Linear SVM

Non linear SVM

Fitting the hyperparameters

Multiclass SVM

Classification of hyperspectral data 


\section{Definition}

Definition (Positive semi-definite kernel) $k: \mathbb{R}^{d} \times \mathbb{R}^{d} \rightarrow \mathbb{R}$ is positive semi-definite is

- $\forall\left(\mathbf{x}, \mathbf{x}^{\prime}\right) \in \mathbb{R}^{d} \times \mathbb{R}^{d}, k\left(\mathbf{x}_{i}, \mathbf{x}_{j}\right)=k\left(\mathbf{x}_{j}, \mathbf{x}_{i}\right)$.

- $\forall n \in \mathbb{N}, \forall \xi_{1} \ldots \xi_{n} \in \mathbb{R}, \forall \mathbf{x}_{1} \ldots \mathbf{x}_{n} \in \mathbb{R}^{d}, \sum_{i, j}^{n} \xi_{i} \xi_{j} k\left(\mathbf{x}_{i}, \mathbf{x}_{j}\right) \geq 0$.

Theorem (Moore-Aronsjan (1950))

To every positive semi-definite kernel $k$, there exists a Hilbert space $\mathcal{H}$ and a feature map $\phi: \mathbb{R}^{d} \rightarrow \mathcal{H}$ such that for all $\mathbf{x}_{i}, \mathbf{x}_{j}$ we have $k\left(\mathbf{x}_{i}, \mathbf{x}_{j}\right)=\left\langle\phi\left(\mathbf{x}_{i}\right), \phi\left(\mathbf{x}_{j}\right)\right\rangle_{\mathcal{H}}$. 
Operations on kernels

Let $k_{1}$ and $k_{2}$ be positive semi-definite, and $\lambda_{1,2}>0$ then:

1. $\lambda_{1} k_{1}$ is a valid kernel

2. $\lambda_{1} k_{1}+\lambda_{2} k_{2}$ is positive semi-definite.

3. $k_{1} k_{2}$ is positive semi-definite.

4. $\exp \left(k_{1}\right)$ is positive semi-definite.

5. $g\left(\mathbf{x}_{i}\right) g\left(\mathbf{x}_{j}\right)$ is positive semi-definite, with $g: \mathbb{R}^{d} \rightarrow \mathbb{R}$. 
Introductory example

Linear case

Non linear case

Concluding remarks

Kernel function

Positive semi-definite kernels

Some kernels

Concluding remarks

Kernel K-NN

Support Vectors Machines

Learn from data

Linear SVM

Non linear SVM

Fitting the hyperparameters

Multiclass SVM

Classification of hyperspectral data 


\section{Polynomial kernel}

The polynomial kernel of order $p$ and bias $q$ is defined as

$$
\begin{aligned}
k\left(\mathbf{x}_{i}, \mathbf{x}_{j}\right) & =\left(\left\langle\mathbf{x}_{i}, \mathbf{x}_{j}\right\rangle+q\right)^{p} \\
& =\sum_{l=1}^{p}\left(\begin{array}{l}
p \\
l
\end{array}\right) q^{p-l}\left\langle\mathbf{x}_{i}, \mathbf{x}_{j}\right\rangle^{\prime} .
\end{aligned}
$$

It correspond to the feature space of monomials up to degree $p$. Depending on $q \gtrless 0$, the relative weights of the higher order monomial is inscreased/deacreased. 


\section{Gaussian kernel}

The Gaussian kernel with paramater $\sigma$ is defined as

$$
k\left(\mathbf{x}_{i}, \mathbf{x}_{j}\right)=\exp \left(-\frac{\left\|\mathbf{x}_{i}-\mathbf{x}_{j}\right\|^{2}}{2 \sigma^{2}}\right) .
$$

More generally, any distance can be used in the exponential rather than the Euclidean distance. For instance, the spectral angle is a valid distance:

$$
\Theta\left(\mathbf{x}_{i}, \mathbf{x}_{j}\right)=\frac{\left\langle\mathbf{x}_{i}, \mathbf{x}_{j}\right\rangle}{\left\|\mathbf{x}_{i}\right\|\left\|\mathbf{x}_{j}\right\|} .
$$




\section{Kernel values in $\mathbb{R}^{2}$}

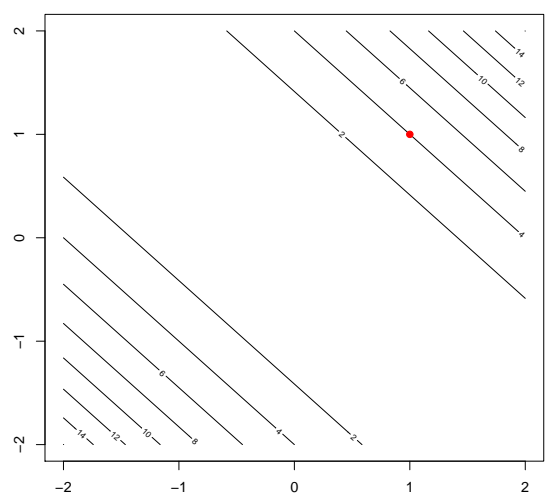

(a)

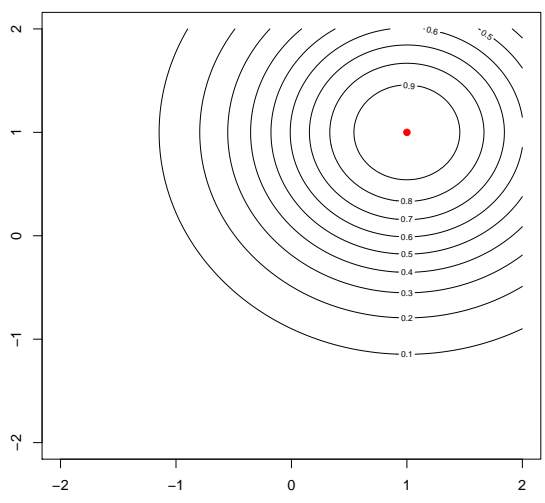

(b)

- (a) Polynomial kernel values for $p=2$ and $q=0$ and $\mathrm{x}=[1,1]$,

- (b) Gaussian kernel values for $\sigma=2$ and $\mathbf{x}=[1,1]$. 
Introductory example

Linear case

Non linear case

Concluding remarks

Kernel function

Positive semi-definite kernels

Some kernels

Concluding remarks

Kernel K-NN

Support Vectors Machines

Learn from data

Linear SVM

Non linear SVM

Fitting the hyperparameters

Multiclass SVM

Classification of hyperspectral data 
How to construct kernel for my data?

- A kernel is usually seen as a measure of similarity between two samples. It reflects in some sens, how two samples are similar.

- In practice, it is possible to define kernels using some a priori of our data.

- For instance: in image classification. It is possible to build kernels that includes information from the spatial domain.

- Local correlation

- Spatial position

$\checkmark \ldots$ 
Introductory example

Linear case

Non linear case

Concluding remarks

Kernel function

Positive semi-definite kernels

Some kernels

Concluding remarks

\section{Kernel K-NN}

Support Vectors Machines

Learn from data

Linear SVM

Non linear SVM

Fitting the hyperparameters

Multiclass SVM

Classification of hyperspectral data

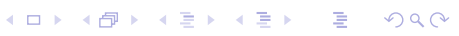




\section{Compute distance in feature space $1 / 2$}

- The K-NN decision rule is based on the distance between two samples. In the feature space, the distance is computed as $\left\|\phi\left(\mathbf{x}_{i}\right)-\phi\left(\mathbf{x}_{j}\right)\right\|_{\mathcal{H}}^{2}$.

- Write this equation in terms of kernel function.

- Fill the function R labwork_knn. R by adding the construction of the kernel function. Then run it. 
Compute distance in feature space $2 / 2$

$$
\begin{aligned}
\left\|\phi\left(\mathbf{x}_{i}\right)-\phi\left(\mathbf{x}_{j}\right)\right\|_{\mathcal{H}}^{2} & =\left\langle\phi\left(\mathbf{x}_{i}\right)-\phi\left(\mathbf{x}_{j}\right), \phi\left(\mathbf{x}_{i}\right)-\phi\left(\mathbf{x}_{j}\right)\right\rangle_{\mathcal{H}} \\
& =\left\langle\phi\left(\mathbf{x}_{i}\right), \phi\left(\mathbf{x}_{i}\right)\right\rangle_{\mathcal{H}}+\left\langle\phi\left(\mathbf{x}_{j}\right), \phi\left(\mathbf{x}_{j}\right)\right\rangle_{\mathcal{H}}-2\left\langle\phi\left(\mathbf{x}_{i}\right), \phi\left(\mathbf{x}_{j}\right)\right\rangle_{\mathcal{H}} \\
& =k\left(\mathbf{x}_{i}, \mathbf{x}_{i}\right)+k\left(\mathbf{x}_{j}, \mathbf{x}_{j}\right)-2 k\left(\mathbf{x}_{i}, \mathbf{x}_{j}\right)
\end{aligned}
$$

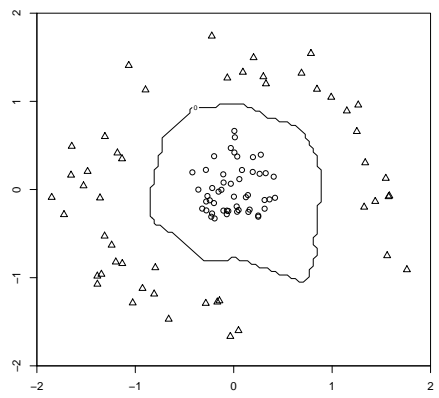

(a)

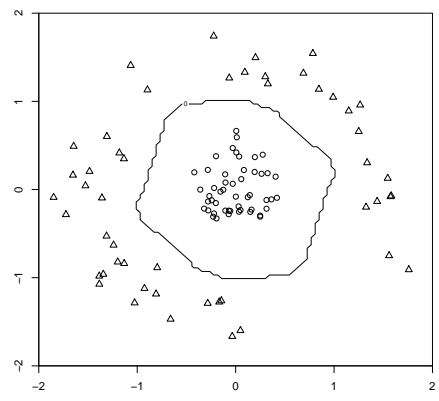

(b)

- (a) KNN classification

- (b) Kernel KNN classification with a polynomial kernel of order 2 
Linear case

Non linear case

Concluding remarks

Kernel function

Positive semi-definite kernels

Some kernels

Concluding remarks

Kernel K-NN

Support Vectors Machines

Learn from data

Linear SVM

Non linear SVM

Fitting the hyperparameters

Multiclass SVM 
Introductory example

Linear case

Non linear case

Concluding remarks

Kernel function

Positive semi-definite kernels

Some kernels

Concluding remarks

Kernel K-NN

Support Vectors Machines

Learn from data

Linear SVM

Non linear SVM

Fitting the hyperparameters

Multiclass SVM

Classification of hyperspectral data 


\section{Learning problem}

- Given a training set $\mathcal{S}$ and a loss function $L$, we want to find a function $f$ from a set of functions $\mathcal{F}$ that minimizes its expected loss, or risk, $R(f)$ :

$$
R(f)=\int_{\mathcal{S}} L(f(\mathbf{x}), y) d \mathcal{P}(\mathbf{x}, y) .
$$

- But $\mathcal{P}(\mathbf{x}, y)$ is unknown!

- The empirical risk, $R_{\text {emp }}(f)$ can be computed:

$$
R_{e m p}(f)=\frac{1}{n} \sum_{i=1}^{n} L\left(f\left(\mathbf{x}^{i}\right), y_{i}\right)
$$

- Convergence?

- $f_{1}$ minimizes $R_{\text {emp }}$, then $R_{e m p}\left(f_{1}\right) \longrightarrow R\left(f_{1}\right)$ as $n$ tends to infinity

- But $f_{1}$ is not necessarily a minimizer of $R$. 
- Bayesian approach consists of selecting a distribution a priori for $\mathcal{P}(\mathbf{x}, y)$ (GMM)

- In machine learning, no assumption is made as to the distribution, but only about the complexity of the class of functions $\mathcal{F}$.

- Favor simple functions to

- discard over-fitting problems,

- to achieve a good generalization ability.

- Vapnik-Chervonenkis (VC) theory: the complexity is related to the number of points that can be separated by a function.

$$
R(f) \leq R_{\text {emp }}(f, n)+\mathcal{C}(f, n)
$$




\section{Illustration}

- Trade-off between $R_{\text {emp }}$ and complexity

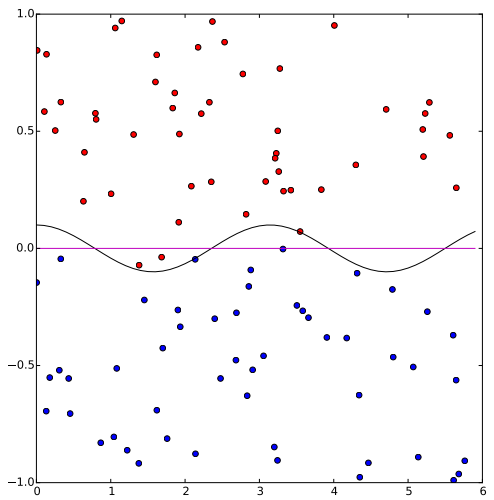

- VC dimension
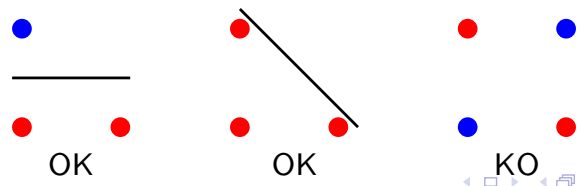
Introductory example

Linear case

Non linear case

Concluding remarks

Kernel function

Positive semi-definite kernels

Some kernels

Concluding remarks

Kernel K-NN

Support Vectors Machines

Learn from data

Linear SVM

Non linear SVM

Fitting the hyperparameters

Multiclass SVM

Classification of hyperspectral data 


\section{Separating hyperplane}

- A separating hyperplane $H(\mathbf{w}, b)$ is a linear decision function that separate the space into two half-spaces, each half-space corresponding to the given class, i.e., $\operatorname{sgn}\left(\left\langle\mathbf{w}, \mathbf{x}_{i}\right\rangle+b\right)=y_{i}$ for all samples from $\mathcal{S}$.

- The condition of correct classification is

$$
y_{i}\left(\left\langle\mathbf{w}, \mathbf{x}_{i}\right\rangle+b\right) \geq 1 .
$$

- Many hyperplanes ?

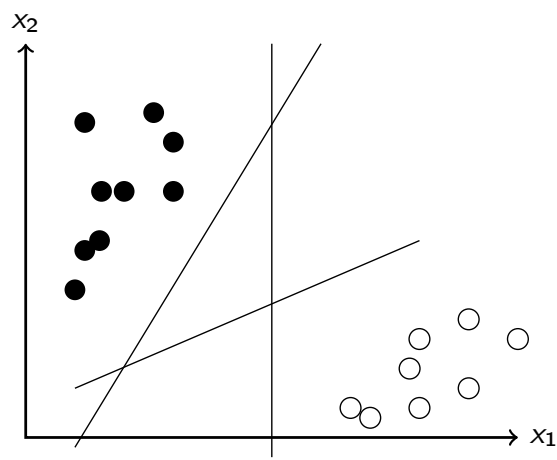




\section{Optimal separating hyperplane}

- From the VC theory, the optimal one is the one that maximize the margin

- The margin is inversely proportional to $\|\mathbf{w}\|^{2}=\langle\mathbf{w}, \mathbf{w}\rangle$.

- Optimal parameters are found by solving the convex optimization problem

$$
\begin{aligned}
& \operatorname{minimize} \frac{\langle\mathbf{w}, \mathbf{w}\rangle}{2} \\
& \text { subject to } y_{i}\left(\left\langle\mathbf{w}, \mathbf{x}_{i}\right\rangle+b\right) \geq 1, \forall i \in 1, \ldots, n .
\end{aligned}
$$

- The problem is traditionally solved by considering soft margin constraints: $y_{i}\left(\left\langle\mathbf{w}, \mathbf{x}_{i}\right\rangle+b\right) \geq 1+\xi_{i}$

$$
\begin{aligned}
& \operatorname{minimize} \frac{\langle\mathbf{w}, \mathbf{w}\rangle}{2}+C \sum_{i=1}^{n} \xi_{i} \\
& \text { subject to } y_{i}\left(\left\langle\mathbf{w}, \mathbf{x}_{i}\right\rangle+b\right) \geq 1-\xi_{i}, \forall i \in 1, \ldots, n \\
& \xi_{i} \geq 0, \forall i \in 1, \ldots, n .
\end{aligned}
$$




\section{Quadratic programming}

- The previous problem is solved by considering the Lagrangian

$$
L(\mathbf{w}, b, \boldsymbol{\xi}, \boldsymbol{\alpha}, \boldsymbol{\beta})=\frac{\langle\mathbf{w}, \mathbf{w}\rangle}{2}+C \sum_{i=1}^{n} \xi_{i}+\sum_{i=1}^{n} \alpha_{i}\left(1-\xi_{i}-y_{i}\left(\left\langle\mathbf{w}, \mathbf{x}_{i}\right\rangle+b\right)\right)-\sum_{i=1}^{n} \beta_{i} \xi_{i}
$$

- Minimizing with respect to the primal variables and maximizing w.r.t the dual variables leads to the so-called dual problem:

$$
\begin{gathered}
\max _{\boldsymbol{\alpha}} g(\boldsymbol{\alpha})=\sum_{i=1}^{n} \alpha_{i}-\frac{1}{2} \sum_{i, j=1}^{n} \alpha_{i} \alpha_{j} y_{i} y_{j}\left\langle\mathbf{x}_{i}, \mathbf{x}_{j}\right\rangle \\
\text { subject to } 0 \leq \alpha_{i} \leq C \\
\sum_{i=1}^{n} \alpha_{i} y_{i}=0 .
\end{gathered}
$$

- $\mathbf{w}=\sum_{i=1}^{n} \alpha_{i} y_{i} \mathbf{x}_{i}$, only some of the $\alpha_{i}$ are non zero. Thus $\mathbf{w}$ is supported by some training samples - those with non-zero optimal $\alpha_{i}$. These are called the support vectors. 
Visual solution of the SVM

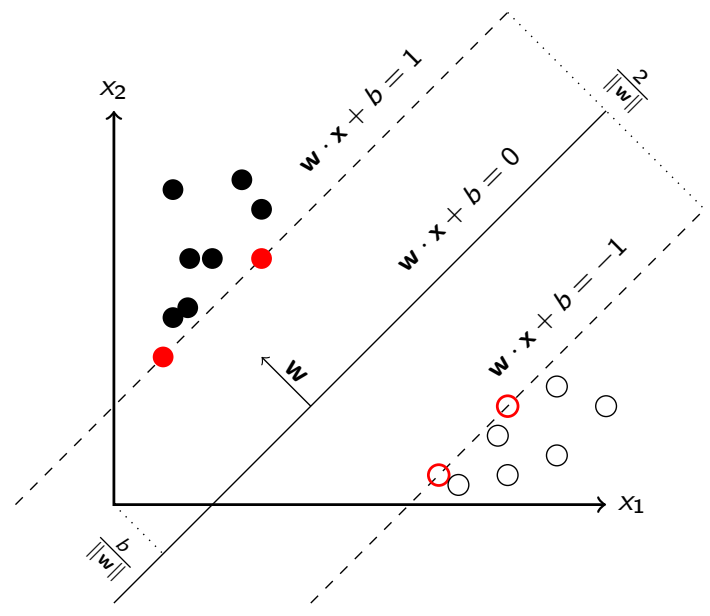


Introductory example

Linear case

Non linear case

Concluding remarks

Kernel function

Positive semi-definite kernels

Some kernels

Concluding remarks

Kernel K-NN

Support Vectors Machines

Learn from data

Linear SVM

Non linear SVM

Fitting the hyperparameters

Multiclass SVM

Classification of hyperspectral data 


\section{Kernelization of the algorithm}

It is possible to extend the linear SVM to non linear SVM by switching the dot product to a kernel function:

$$
\begin{gathered}
\max _{\boldsymbol{\alpha}} g(\boldsymbol{\alpha})=\sum_{i=1}^{n} \alpha_{i}-\frac{1}{2} \sum_{i, j=1}^{n} \alpha_{i} \alpha_{j} y_{i} y_{j} k\left(\mathrm{x}_{i}, \mathrm{x}_{j}\right) \\
\text { subject to } 0 \leq \alpha_{i} \leq \mathrm{C} \\
\sum_{i=1}^{n} \alpha_{i} y_{i}=0 .
\end{gathered}
$$

Now, the SVM is a non-linear classifier in the input space $\mathbb{R}^{d}$, but is still linear in the feature space - the space induced by the kernel function. The decision function is simply:

$$
f(\mathbf{x})=\operatorname{sgn}\left(\sum_{i=1}^{n} \alpha_{i} y_{i} k\left(\mathbf{x}, \mathbf{x}_{i}\right)+b\right)
$$


Toy example with the Gaussian kernel
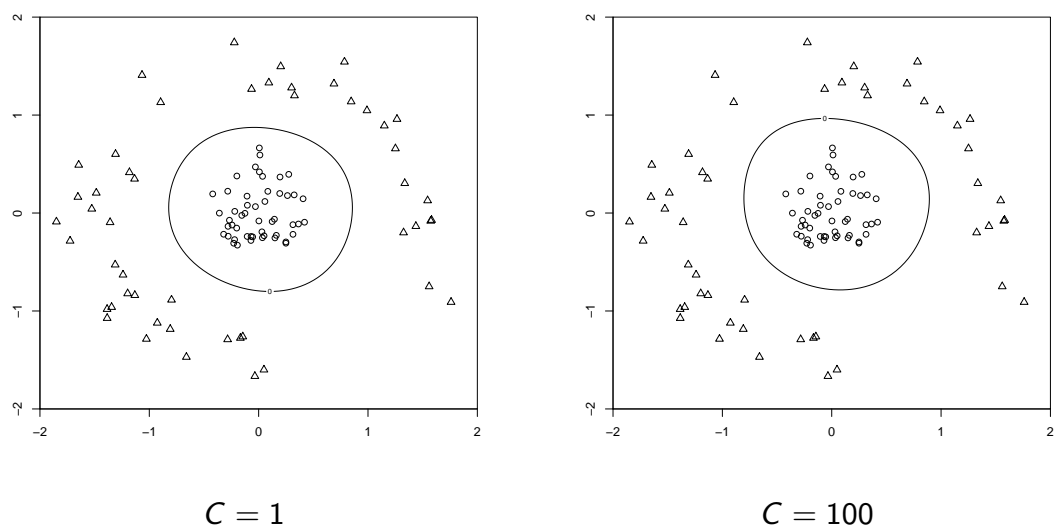


\section{Comparison with GMM}

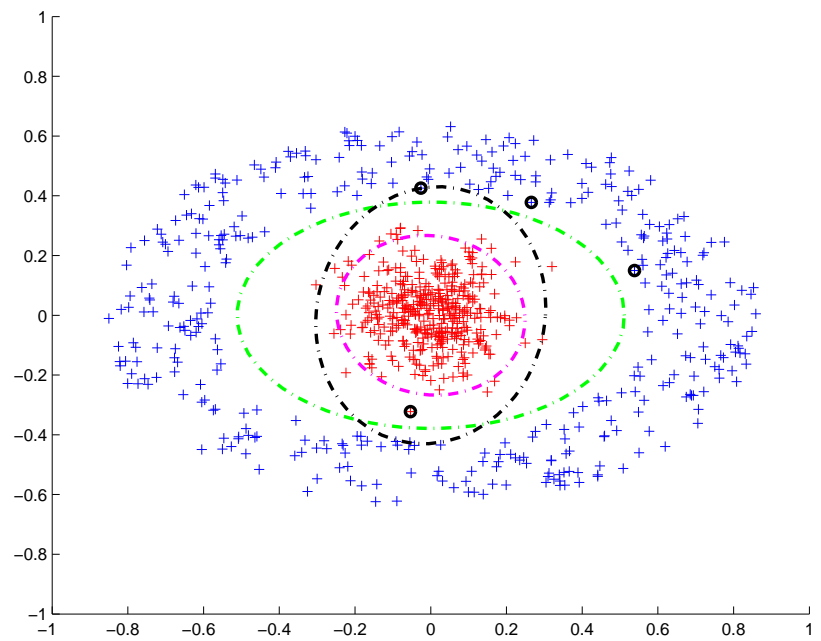


Introductory example

Linear case

Non linear case

Concluding remarks

Kernel function

Positive semi-definite kernels

Some kernels

Concluding remarks

Kernel K-NN

Support Vectors Machines

Learn from data

Linear SVM

Non linear SVM

Fitting the hyperparameters

Multiclass SVM

Classification of hyperspectral data 


\section{Cross-validation}

- Crucial step: improve or decrease drastically the performances of SVM

- Cross validation is conventionally used. CV estimates the expected error $R$.

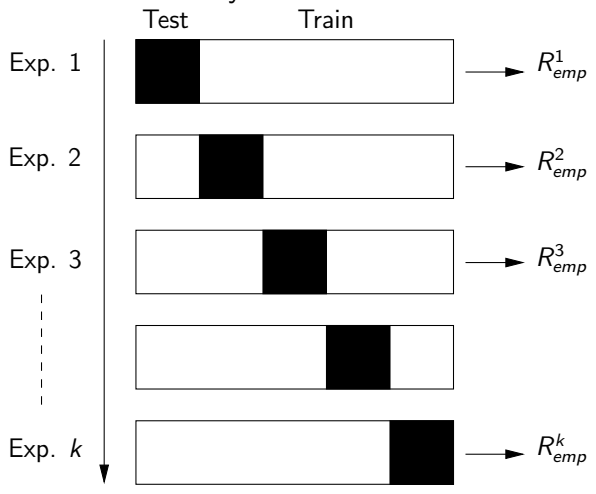

- $R(\mathbf{p}) \approx \frac{1}{k} \sum_{i=1}^{k} R_{e m p}^{i}$

- Good behavior in various supervised learning problem but high computational load. Test 10 values with $k=5 \Rightarrow \mathbf{5 0}$ learning steps. But it can be perform in parallel... 
Introductory example

Linear case

Non linear case

Concluding remarks

Kernel function

Positive semi-definite kernels

Some kernels

Concluding remarks

Kernel K-NN

Support Vectors Machines

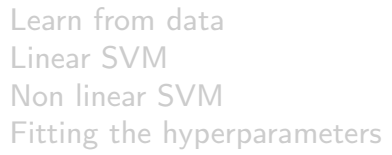

Multiclass SVM

Classification of hyperspectral data 
Collection of binary classifiers

- One versus All: $m$ binary classifiers

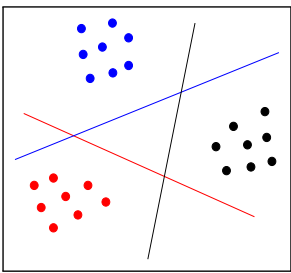

- One versus One: $\mathrm{m}(\mathrm{m}-1) / 2$ classifiers
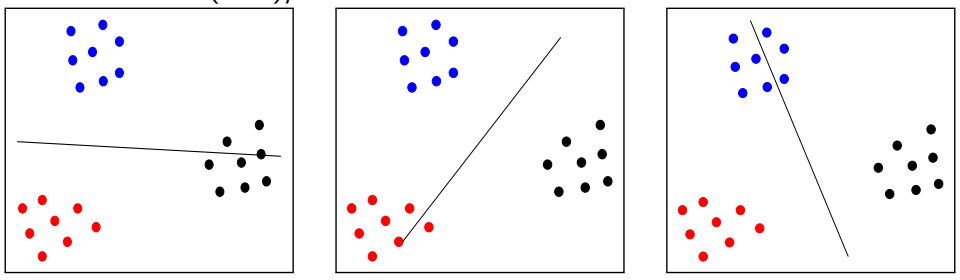
Introductory example

Linear case

Non linear case

Concluding remarks

Kernel function

Positive semi-definite kernels

Some kernels

Concluding remarks

Kernel K-NN

Support Vectors Machines

Learn from data

Linear SVM

Non linear SVM

Fitting the hyperparameters

Multiclass SVM

Classification of hyperspectral data 
- Run the code toy_svm.R

- The default classification is done with a Gaussian kernel: try to do it with a polynomial kernel

- Check the influence of each hyperparameters for the Gaussian and Polynomial kernel

- For the Gaussian kernel and a given set of hyperparameters

- Get the number of support vectors

- Plot them

- Conclusions? 


\section{Simulated data}

- Simulated reflectance of Mars surface (500 to $5200 \mathrm{~nm}$ )

- The model has 5 parameters (Sylvain Douté): the grain size of water and $\mathrm{CO}_{2}$ ice, the proportion of water, $\mathrm{CO}_{2}$ ice and dust.

- $\mathrm{x} \in \mathbb{R}^{184}$ and $n=31500$.

- Fives classes according to the grain size of water.

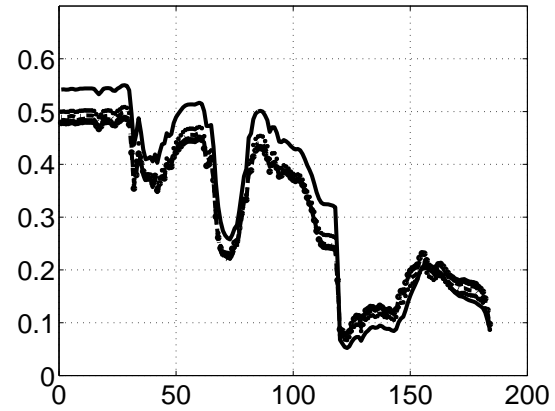

In this labwork, we are going to use the $\mathrm{R}$ package e1071 that use the $\mathrm{C}++$ library libsvm, the state of the art QP solver. 


\section{Questions}

- Using the file main_svm.R, classify the data set with

- SVM with a Gaussian kernel,

- K-NN and Kernel KNN (with a polynomial kernel)

- Implement the cross-validation for SVM, to select the optimal hyperparameters $(\mathrm{C}, \sigma)$

- Compute the confusion matrix for each methods and look at the classification accuracy 


\section{Load the data}

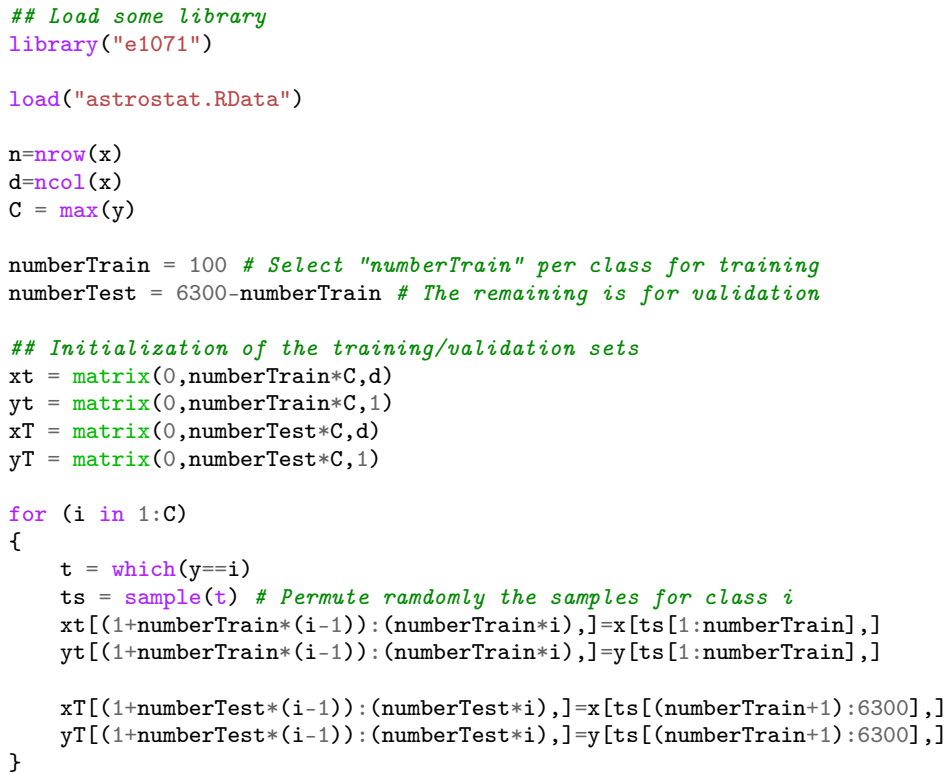




\section{Perform a simple classification}

\#\# Learn the model

model $=\operatorname{svm}(x t, y t, \cos t=1$, gamma $=0.001$, type $=" C "$, cachesize $=512)$

\#\# Predict the validation samples

$\mathrm{yp}=\operatorname{predict}(\operatorname{model}, \mathrm{xT})$

\#\# Confusion matrix

confu $=$ table $(y \mathrm{~T}, \mathrm{yp})$

$\mathrm{OA}=\operatorname{sum}(\operatorname{diag}(\operatorname{confu})) / \operatorname{sum}(\operatorname{confu})$ 